\title{
Prevención y tratamiento de las infecciones del sitio operatorio en neurocirugía. Estado del arte
}

\author{
Julio César García-Casallas', Jhósep Andrés Blanco-Mejía², Yuli Viviana Fuentes-Barreiro ${ }^{3}$, \\ Laura Camila Arciniegas-Mayorga ${ }^{4}$, César Daniel Arias-Cepeda ${ }^{5}$, Brayan David Morales-Pardo ${ }^{6}$
}

\section{RESUMEN}

El manejo adecuado de las infecciones del sitio operatorio (ISO) en neurocirugía es fundamental para la disminución de la carga de morbilidad y mortalidad en estos pacientes. La sospecha y confirmación diagnóstica asociadas al aislamiento microbiológico son esenciales para asegurar el tratamiento oportuno y el adecuado gerenciamiento de antibióticos. En esta revisión se presenta de forma resumida los puntos fundamentales para la prevención y el tratamiento de infecciones del sitio operatorio en neurocirugía y se incluye un apartado sobre el uso de antibióticos intratecales/intraventriculares.

\section{PALABRAS CLAVE}

Infección de la Herida Quirúroica; Infecciones del Sistema Nervioso Central; Prevención de Enfermedades; Procedimientos Neuroquirúroicos; Profilaxis Antibiótica; Terapéutica; Traumatismos Craneoencefálicos

\section{SUMMARY}

\section{Prevention and treatment of surgical site infections in neurosurgery: state of the art}

The adequate management of surgical wound infections in neurosurgery is fundamental for reducing the burden of morbidity and mortality in these patients. The suspicion and diagnostic

\footnotetext{
1 Director de programa Facultad de Medicina, Universidad de La Sabana. Director de departamento Farmacología clínica, Clínica Universidad de La Sabana. Bogotá D.C, Colombia.

2 Médico general, Hospital Manuel Elkin Patarroyo. Inírida-Guainía, Colombia.

Médico general, Grupo de Investigación en Cirugía de Columna, Fundación Santa Fe de Bogotá. Bogotá D.C, Colombia.

Médico general, Hospital San Vicente de Paúl. Nemocón-Cundinamarca, Colombia.

Médico general, Hospital San Antonio de Sesquilé. Sesquilé-Cundinamarca, Colombia.

Médico general, Hospital San José de Guaduas. Guaduas-Cundinamarca, Colombia.

Correspondencia: Julio César García Casallas; julio.garcia@unisabana.edu.co

Recibido: julio 15 de 2018

Aceptado: octubre 12 de 2018
}

Cómo citar: García-Casallas JC, Blanco-Mejía JA, Fuentes-Barreiro YV, Arciniegas-Mayorga LC, Arias-Cepeda CD, Morales-Pardo BD. Prevención y tratamiento de las infecciones del sitio operatorio en neurocirugía. Estado del arte. latreia. 2020 Ene-Mar;33(1):39-58. D0I 10.17533/udea.iatreia.23. 
confirmation associated with microbiological isolation are essential to ensure timely treatment and proper management of antibiotics. Therefore, in this review we present, in a synthetic manner, the main points for the prevention and treatment of surgical site infections in neurosurgery, which includes a section on the use of intrathecal/intraventricular antibiotics.

\section{KEY WORDS}

Antibiotic Prophylaxis; Central Nervous System Infections; Craniocerebral Traumas; Disease Prevention; Neurosurgical Procedures; Surgical Wound Infections; Therapeutics

\section{INTRODUCCIÓN}

Las ISO en neurocirugía son una amenaza para el éxito de las intervenciones quirúrogicas; se asocian con una alta carga de morbilidad, mortalidad y costos para el paciente neuroquirúrogico ${ }^{(1-4)}$. El conocimiento para la prevención y el tratamiento de este tipo de infecciones es fundamental, bajo la premisa de que se convierten en un reto terapéutico por la complejidad de las intervenciones y por las condiciones anatómicas y microscópicas del sistema nervioso central ${ }^{(\mathrm{SNC})}{ }^{(1-4)}$. Lo anterior se asocia con la problemática global de la multirresistencia a los antibióticos, así como con la necesidad de hacer un gerenciamiento adecuado de los antimicrobianos ${ }^{(5)}$.

La tasa de las ISO en neurocirugía es variable, depende del procedimiento quirúrgico ${ }^{(6,7)}$, del uso de dispositivos médicos y su duración ${ }^{(8-16)}$, del tiempo quirúroico ${ }^{(17-19)}$, de las características propias de los pacientes y de su enfermedad ${ }^{(17)}$, de la preparación de la piel ${ }^{(17-19)}$, de la intervención del personal en formación ${ }^{(19)}$, de la ubicación geográfica ${ }^{(8,9,13,16)}$ y de la contaminación directa o la presencia de fistulas de líquido cefalorraquídeo (LCR) en el paciente con trauma craneoencefálico (TCE) ${ }^{(6-19)}$. De la misma manera, los microorganismos reportados pueden ser variables. Los más comunes son Staphylococcus aureus, con énfasis en el meticilino resistente (SAMR), Staphylococcus coagulasa negativos; bacilos gramnegativos y anaerobios como el Cutibacterium acnes (antes Propionibacterium acnes), este último especialmente relacionado con la colocación de dispositivos médicos y material de osteosíntesis ${ }^{(6,7,10,14)}$.

\section{EPIDEMIOLOGÍA}

La ocurrencia de las ISO en neurocirugía puede ser menor al $1 \%$ y tan alta como el $41 \%{ }^{(6-19)}$. En un informe del Centers for Disease Control and Prevention (CDC) de Estados Unidos, se menciona la incidencia de infección en cuatro procedimientos de neurocirugía: craneotomía 2,6\%, espondilosindesis 1,5\%, laminectomía 1 \% y derivación ventricular de LCR 5,6 \% ${ }^{(9)}$. Otros estudios de la Comunidad Científica Internacional de Control de Infecciones Nosocomiales (INICC), que incluyó a 30 países en vía de desarrollo, entre ellos Colombia y otros países de Latinoamérica, encontró tasas más altas y riesgos relativos más elevados de las ISO en estos mismos procedimientos (craneotomía $4,4 \%$, espondilosindesis 3,2 \%, laminectomía 1,7 \% y drenaje ventricular 12,9\%), comparados con el informe del CDC (16). McClelland reportó la tasa de infección del sitio operatorio en una cirugía intracraneal de 2,2 \% en Estados Unidos y, de 5,7 \% en Europa ${ }^{(13)}$.

En Colombia, un estudio encontró la tasa de infección en espondilosindesis del 2,6 \% y en laminectomía del $9,1 \%$, siendo este último procedimiento el de mayor incidencia de infección y el que más se alejaba de los reportes epidemiológicos internacionales ${ }^{(8)}$.

En las series revisadas por la Infectious Diseases Society of America (IDSA), las derivaciones del líquido cefalorraquídeo (LCR) mostraron un rango promedio de ISO entre el 4-17 \%; entre el 3,6-20 \% con el uso de bombas de infusión, y para los dispositivos de estimulación cerebral profunda entre 0,62-14,3 \% ${ }^{(7)}$. En drenes ventriculares externos la incidencia encontrada fue entre 0-22\%, con tasas de 11,4 por cada 1000 días con el catéter ${ }^{(7,12,15)}$. Para los drenes lumbares externos las ISO se ubican en un $5 \%$ de ocurrencia, pero puede ser tan baja como del 0,8 \% ${ }^{(11)}$. Finalmente, en el caso de las infecciones en la cirugía de columna, el rango de infección varía entre el 1-14 \% con incidencia aproximada entre el 2-8,5\% cuando existe instrumentación o colocación de prótesis artificial de disco intervertebral ${ }^{(10,14)}$.

\section{PREVENCIÓN DE LA INFECCIÓN DEL SITIO OPERATORIO}

Las investigaciones en este punto se centran en el uso de protocolos estandarizados para el 
posicionamiento, manutención y manipulación de drenes y derivaciones; en la ađministración profiláctica de antibióticos sistémicos y/o tópicos; en el uso de derivaciones y drenajes impregnados de antibiótico o iones de plata y en evitar el cambio de drenes externos en intervalos fijos ${ }^{(7,20)}$. Cabe mencionar la efectividad de las medidas generales de prevención, como el lavado de manos, el control de la glicemia del paciente, el mantenimiento del aire limpio en el quirófano, la implementación rigurosa de protocolos de asepsia y antisepsia para la preparación del paciente, el uso de instrumental estéril y la disciplina del equipo quirúrgico ${ }^{(18)}$.

\section{PROTOCOLOS DE ATENCIÓN}

Los protocolos quirúrgicos integrales, que incluyen los protocolos de mínima manipulación de dispositivos médicos en los diferentes estudios, han mostrado una reducción de la tasa de ISO y del recambio de dispositivos, pese a los diferentes rangos de adhesión del personal de salud ${ }^{(20)}$. Las intervenciones que incluyen los protocolos son: uso de suturas impregnadas de antibiótico, política del no rasurado, uso de doble guante, cambio de guantes previamente a la manipulación de dispositivos, lavado del cabello con clorhexidina al $5 \%$, tunelización subcutánea extensa con colocación de apósitos de hidrocoloides para la protección de las suturas de fijación del dispositivo, mínima manipulación de los dispositivos, toma de muestras de LCR cuando es estrictamente necesario y recolección de LCR por goteo y no por aspirado, para evitar la obstrucción del sistema ${ }^{(20,21-24)}$.

\section{CATÉTERES IMPREGNADOS CON ANTIBIÓTICOS O IONES DE PLATA}

Las revisiones sistemáticas disponibles favorecen su utilización, sin diferenciar entre los recubiertos por clindamicina o minociclina con rifampicina, al encontrar reducción neta estadísticamente significativa del riesgo de infección. Lo anterior es aplicable a todos los grupos de edad evaluados (neonatos, población pediátrica y adulta) con una reducción más evidente en la infección causada por Staphylococcus spp ${ }^{(25-33)}$. No obstante, también existen estudios que no encuentran diferencia en la ocurrencia de infección con el uso de estos dispositivos y no recomiendan su uso ${ }^{(34,35)}$, aunque la IDSA los recomienda ${ }^{(7)}$. En las infecciones tardías ( $>6$ meses de colocación del sistema de derivación) los metaanálisis presentados no encuentran diferencias significativas; sin embargo, existen estudios que concluyen que los catéteres impregnados de antibiótico no aumentan el riesgo y sí podrían retrasar la aparición de una infección en los pacientes con hemorragia subaracnoidea que requieren ventriculostomía ${ }^{(36,37)}$.

De igual manera, la evidencia aún no es concluyente sobre el uso de catéteres impregnados y profilaxis sistémica prolongada conjunta ${ }^{(38,39)}$. Igualmente, frente al uso de catéteres impregnados de iones de plata, los estudios revisados han encontrado una reducción en las infecciones del sitio operatorio de forma significativa. Sin embargo, no es conclusiva en subgrupos de gérmenes causales ${ }^{(27,40)}$. Con respecto a la inyección de antibióticos (4 mg de gentamicina +10 mg de vancomicina en $2 \mathrm{ml}$ de solución salina normal) en el reservorio de los sistemas de derivación, algunos estudios han encontrado un beneficio, no obstante, aún se requieren más investigaciones sobre esta intervención ${ }^{(41,42)}$.

\section{ANTIBIÓTICOS SISTÉMICOS PROFILÁCTICOS}

El uso de antibiótico profiláctico sistémico es controversial; sin embargo, la evidencia actual halla un efecto benéfico de su administración con la reducción de las tasas de infección postoperatoria, que varía desde un $20 \%$ hasta un $50 \%{ }^{(43)}$. Igualmente, sugiere la fuga de LCR como el principal factor de riesgo para la infección, por lo que si persiste por más de 7 días debería considerarse el manejo quirúrgico $(31,44-51)$. En intervenciones quirúrgicas como el drenaje ventricular externo se requiere ampliar la evidencia, especialmente en sí es útil la ađministración continúa hasta el retiro del catéter ${ }^{(31,49)}$. Igualmente, en cirugía de base de cráneo, la administración de profilaxis no mostró una diferencia significativa en la tasa de meningitis posoperatoria ${ }^{(52)}$.

Los regímenes de antibióticos usados en los diferentes estudios no son homogéneos. Dentro de estos, se 
incluyen el uso de vancomicina, oxacilina, clindamicina, cefazolina, trimetoprim sulfametoxazol, ampicilina y cefalosporinas de tercera generación, los cuales cumplen fundamentalmente con el cubrimiento de microorganismos grampositivos, teniendo en cuenta que el principal agente involucrado es el Staphylococcus spp ${ }^{(31,44-52,53)}$. Algunos estudios han favorecido el uso de vancomicina, especialmente en contextos de alta prevalencia de SAMR, pero otros no han encontrado diferencias entre esquemas ${ }^{(53,54,55)}$. Se concluye así, que aún falta evidencia para determinar qué régimen antibiótico es el más óptimo ${ }^{(44,49,52-55)}$. La literatura y las guías de manejo actuales recomiendan la administración de antimicrobianos previamente a la incisión (1 hora antes) y no extender su uso por más de 24 horas del postoperatorio, debido al riesgo de incrementar la resistencia antibiótica ${ }^{(7,44,49)}$.

\section{ANTIBIÓTICOS TÓPICOS}

El uso directo de la vancomicina en polvo en la herida antes de cerrar los planos quirúroicos se fundamenta en el alcance de la concentración inhibitoria mínima (CIM) de forma rápida, lo que minimizaría la concentración sérica necesaria; lo anterior presentaría como ventaja la disminución de efectos adversos como la nefrotoxicidad reportada en el uso sistémico ${ }^{(56)}$.

En la actualidad, se encuentran más estudios relacionados con el uso de la vancomicina tópica en la cirugía de columna que de otros tipos de procedimientos neuroquirúroicos, estos favorecen su uso en la cirugía de columna instrumentada y no instrumentada, craneotomía, implantación de estimulador cerebral profundo y colocación de derivaciones de LCR ${ }^{(57-68)}$; no obstante, su uso aún constituye una intervención experimental, si bien, al parecer, es segura con una baja tasa general de reacciones adversas $(0,3 \%)^{(56-69)}$.

\section{PROFILAXIS ANTIBIÓTICA EN EL TRAUMA CRANEOENCEFÁLICO}

La profilaxis se ha evaluado considerando las características diferenciales del trauma como lo son el tipo y lugar del trauma, exposición de masa encefálica, trauma penetrante, presencia de fístulas de LCR, fracturas de la base del cráneo o en comunicación con los senos paranasales ${ }^{(49,70,71)}$. En el metaanálisis realizado por Poole et al., se evaluó el uso de antibióticos profilácticos en fracturas de base de cráneo, estudio en el cual no se demostró beneficio aparente en la instauración de esta medida ${ }^{(71)}$.

Una revisión de Cochrane evaluó pacientes con fractura traumática de base de cráneo asociado o no a fístula de LCR, la conclusión es que la evidencia disponible es de calidad dudosa y no soporta el uso profiláctico de corta o larga duración en estos casos, independientemente de la existencia de fístulas de LCR ${ }^{(49)}$.

Adicionalmente, en los pacientes con técnicas invasivas de monitorización no se ha encontrado evidencia a favor de la instauración de ciclos de corta o larga duración de antimicrobianos para la reducción de estas infecciones ${ }^{(70)}$. Por otra parte, un ensayo clínico con uso de ceftriaxona profiláctica para pacientes con neumoencéfalo tampoco demostró diferencias significativas ${ }^{(72)}$.

\section{INFECCIÓN INCISIONAL SUPERFICIAL}

El riesgo de infección del sitio operatorio en los procedimientos neuroquirúrgicos limpios es de 4 a $12 \%$ en algunos metaanálisis ${ }^{(73-75)}$. Los factores de riesgo para la infección del sitio operatorio posterior a la craneotomía son la fistula de LCR, aspiración de LCR, manipulación de senos venosos, el tiempo quirúrgico mayor a 4 horas, cirugía por causas no traumáticas y clasificación ASA $>2^{(73-75)}$. Por otra parte, en cirugía de columna existen otros factores de riesgo como la diabetes mal controlada, el abordaje quirúrogico posterior, el tiempo quirúroico mayor a 3 horas, la obesidad mórbida (IMC > 35) y el número de niveles intervertebrales intervenidos (mayor a 7$)^{(73-75)}$. Los regímenes antimicrobianos para la ISO superficial son similares a los de otras heridas quirúrogicas limpias, teniendo en cuenta que la principal medida terapéutica es el desbridamiento de la herida y la curación por segunda intención ${ }^{(76,77)}$. Asimismo, debe pensarse en el cubrimiento con $\beta$-lactámicos antiestafilocócicos o, en el caso de sospechar de Staphylococcus resistentes a la meticilina, iniciar vancomicina de 5 a 7 días ${ }^{(76}$. 77) (Tabla 1 y 2). 
Tabla 1. Manejo antibiótico dirigido de ISO superficial y profunda

\begin{tabular}{|c|c|}
\hline Sospecha o aislamiento microbiológico & Antimicrobianos de elección \\
\hline Staphylococcus meticilino sensible ${ }^{*} \dagger$ & $\begin{array}{l}\text { Oxacilina o cefazolina } \\
\text { Alternativas: vancomicina, clindamicina, trimetroprim sulfametoxazol }\end{array}$ \\
\hline Staphylococcus meticilino resistente ${ }^{*} \dagger$ & $\begin{array}{l}\text { Vancomicina } \\
\text { Alternativas: linezolid, daptomicina, trimetroprim sulfametoxazol, } \\
\text { evaluar clindamicina } \neq\end{array}$ \\
\hline Streptococcus pneumoniae sensible a la penicilina†' $\S$ & $\begin{array}{l}\text { Penicilina G } \\
\text { Alternativas: cefotaxime o ceftriaxona, clindamicina }\end{array}$ \\
\hline $\begin{array}{l}\text { Streptococcus pneumoniae resistente a la penicilina y } \\
\text { sensible a las cefalosporinas de 3ra generación } \uparrow \S\end{array}$ & $\begin{array}{l}\text { Cefotaxime o ceftriaxona } \\
\text { Alternativas: cefepime, vancomicina, moxifloxacino, clindamicina }\end{array}$ \\
\hline $\begin{array}{l}\text { Streptococcus pneumoniae resistente a la penicilina y } \\
\text { resistente a las cefalosporinas de 3ra generación }+\S\end{array}$ & $\begin{array}{l}\text { Cefotaxime o ceftriaxona más vancomicina } \\
\text { Alternativas: cefepime + vancomicina, moxifloxacino, clindamicina. }\end{array}$ \\
\hline Haemophilus influenzae no productora de B-lactamasas & $\begin{array}{l}\text { Ampicilina } \\
\text { Alternativas: cefotaxime, ceftriaxona, cefepime, fluoroquinolonas }\end{array}$ \\
\hline Haemophilus influenzae productora de B-lactamasas & $\begin{array}{l}\text { Cefotaxime o ceftriaxona } \\
\text { Alternativas: cefepime, aztreonam, fluoroquinolona }\end{array}$ \\
\hline Cutibacterium acnes & $\begin{array}{l}\text { Penicilina G } \\
\text { Alternativas: clindamicina, cefalosporinas de 3ra generación, vanco- } \\
\text { micina, linezolid, daptomicina }\end{array}$ \\
\hline Enterobacterias sin AMP-C cromosómico de patrón usual & $\begin{array}{l}\text { Cefazolina } \\
\text { Alternativas: cefotaxime, o ceftriaxona, fluoroquinolona, trimetro- } \\
\text { prim sulfametoxazol } \|\end{array}$ \\
\hline $\begin{array}{l}\text { Bacilos gramnegativos con AMP-C cromosómico (No } \\
\text { incluye Pseudomonas spp. ni Acinetobacter spp.) }\end{array}$ & $\begin{array}{l}\text { Cefepime } \\
\text { Alternativas: ertapenem, meropenem, fluoroquinolonas, trimetro- } \\
\text { prim sulfametoxazol || }\end{array}$ \\
\hline $\begin{array}{l}\text { Bacilos gramnegativos productores de B-lactamasas de } \\
\text { espectro extendido (BLEE) }\end{array}$ & $\begin{array}{l}\text { Ertapenem } \\
\text { Alternativas: meropenem, fluoroquinolonas, trimetroprim sulfame- } \\
\text { toxazol || }\end{array}$ \\
\hline Pseudomonas auruginosa & $\begin{array}{l}\text { Cefepime } \\
\text { Alternativas: ceftazidime, piperacilina tazobactam, meropenem, } \\
\text { ciprofloxacino, colistina o polimixina B, aztreonam. }\end{array}$ \\
\hline Acinetobacter baumannii & $\begin{array}{l}\text { Meropenem } \\
\text { Alternativas: colistina o polimixina B }\end{array}$ \\
\hline Bacilos gramnegativos productoras de carbapenemasas & $\begin{array}{l}\text { Combinación de dos o más agentes: colistina o polimixina B, dori- } \\
\text { penem o meropenem, tigeciclina, amikacina si no es SNC (pensar } \\
\text { intratecal) }\end{array}$ \\
\hline Candida spp. & $\begin{array}{l}\text { Equinocandinas } \\
\text { Alternativas: fluconazol, voriconazol, anfotericina B }\end{array}$ \\
\hline Aspergillus spp. & $\begin{array}{l}\text { Voriconazol } \\
\text { Alternativas: anfotericina B liposomal o posaconazol }\end{array}$ \\
\hline
\end{tabular}

ISO: infección del sitio operatorio. SNC: sistema nervioso central. spp: especies. * Incluye Staphylococcus aureus y otras especies coagulasa positiva o negativa. † Considerar adicionar o, como alternativa en la monoterapia la rifampicina. ‡ En Staphylococcus aureus adquirido en la comunidad sin gen erm. $\S$ Usar puntos de corte de concentración inhibitoria mínima, diferenciales del sistema nervioso central o de otros tejidos según corresponda. || Solo en el tratamiento dirigido con conocimiento de sensibilidad. Fuente: creación propia 
Tabla 2. Manejo antibiótico dirigido de ISO órgano-espacio (SNC)

\section{Sospecha o aislamiento microbiológico}

Staphylococcus meticilino sensible * $†$

Staphylococcus meticilino resistente ${ }^{*} \dagger$

Streptococcus pneumoniae sensible a la penicilina $\uparrow$ `

Streptococcus pneumoniae resistente a la penicilina y sensibilidad a las cefalosporinas de 3ra generación + , $\S$

Streptococcus pneumoniae resistente a la penicilina y resistencia a las cefalosporinas de 3ra generación †’

Haemophilus influenzae no productora de B-lactamasas

Haemophilus influenzae productora de B-lactamasas

Cutibacterium acnes

Enterobacterias sin AMP-C cromosómico de patrón usual

Bacilos gramnegativos con AMP-C cromosómico (No incluye Pseudomonas spp. ni Acinetobacter spp.)

Bacilos gramnegativos productores de B-lactamasas de espectro extendido (BLEE)

Pseudomonas auruginosa

Acinetobacter baumannii

Bacilos gramnegativos productoras de carbapenemasas

Candida spp.

Aspergillus spp.

\section{Antimicrobianos de elección}

Oxacilina

Alternativas: vancomicina, linezolid, daptomicina, trimetroprim sulfametoxazol

Vancomicina

Alternativas: linezolid, daptomicina, trimetroprim sulfametoxazol

Penicilina G

Alternativas: cefotaxime o ceftriaxona

Cefotaxime o ceftriaxona

Alternativas: cefepime, vancomicina, moxifloxacino.

Cefotaxime o ceftriaxona más vancomicina

Alternativas: cefepime + vancomicina, meropenem, moxifloxacino.

Ampicilina

Alternativas: cefotaxime, ceftriaxona, cefepime, fluoroquinolonas

Cefotaxime o ceftriaxona

Alternativas: cefepime, aztreonam, fluoroquinolona

Penicilina G

Alternativas: cefalosporinas de 3ra generación, vancomicina, linezolid, daptomicina

Ceftriaxona o cefotaxime

Alternativas: cefepime, fluoroquinolona, meropenem, trimetroprim sulfametoxazol \||

Meropenem

Alternativas: fluoroquinolonas, colistina o polimixina B, trimetroprim sulfametoxazol ||

Meropenem

Alternativas: fluoroquinolonas, colistina o polimixina B, trimetroprim sulfametoxazol \||

Cefepime

Alternativas: ceftazidime, meropenem, ciprofloxacino, colistina o polimixina $\mathrm{B}$, aztreonam.

Meropenem

Alternativas: colistina o polimixina B

Combinación de dos o más agentes: colistina o polimixina $B$, doripenem o meropenem, tigeciclina, amikacina si no es SNC (pensar intratecal)

Anfotericina B liposomal con o sin fluocitosina Alternativas: fluconazol o voriconazol

Voriconazol

Alternativas: anfotericina B liposomal o posaconazol

ISO: infección del sitio operatorio. SNC: sistema nervioso central. spp: especies. * Incluye Staphylococcus aureus y otras especies coagulasa positiva o negativa. † Considerar adicionar o, como alternativa en la monoterapia la rifampicina. ‡ En Staphylococcus aureus adquirido en la comunidad sin gen erm. $\S$ Usar puntos de corte de concentración inhibitoria mínima, diferenciales del sistema nervioso central o de otros tejidos según corresponda. || Solo en el tratamiento dirigido con conocimiento de sensibilidad. Fuente: creación propia 


\section{INFECCIÓN INCISIONAL PROFUNDAY OSTEOMIELITIS VERTEBRAL POSOPERATORIA}

El diagnóstico es fundamentalmente clínico, por lo cual es necesario determinar la profundidad de la infección, el tipo de estructuras comprometidas y el tiempo de presentación (menor o mayor a 90 días) (78-87). La principal manifestación clínica en las infecciones tempranas es el dolor desproporcionado en el sitio intervenido, asociado o no a fiebre, secreción, eritema e induración; y pseudoartrosis o aflojamiento del material de osteosíntesis en el caso de las infecciones latentes ${ }^{(82-84,86)}$. Por otro lado, los niveles elevados de reactantes de la fase aguda pueden atribuirse al procedimiento en las primeras 2 o 6 semanas en el caso de la PCR y VSG, respectivamente ${ }^{(82-84,86)}$.

Se debe anotar que los cultivos superficiales o de la secreción son poco específicos, por lo que las muestras tomadas intraoperatoriamente representan el examen diagnóstico de elección ${ }^{(78-80,82-87,88,89)}$. La radiografía puede mostrar cambios sutiles tales como el aflojamiento del material y las irregularidades en la integridad vertebral ${ }^{(78-80,82-89,90-92) .}$ Otras imágenes más sensibles y específicas como la resonancia magnética o la gammagrafía con leucocitos marcados, son muy útiles para el diagnóstico ${ }^{(78-80,82-92)}$.

Para el manejo de la ISO, las indicaciones encontradas para reintervención son la progresión de la infección a pesar del manejo antibiótico, el compromiso del canal espinal con sionos de focalización neurológica o la destrucción progresiva de los cuerpos vertebrales con deformidad ${ }^{(88-90)}$. En el caso de identificarse colecciones, el tratamiento de elección es el lavado y drenaje quirúrogico, asociado a antibioticoterapia endovenosa por alrededor de 6 semanas de manera dirigida por aislamiento microbiológico, luego de punciones aspirativas del disco o hasta las 8 semanas en los casos de SAMR ${ }^{(82-84,86,88-90)}$ (Tabla 1 y 2). Los expertos, en general, prefieren preservar el instrumental cuando sea posible, especialmente en las infecciones tempranas (menos de 90 días), a excepción de que se encuentre suelto o su retiro no represente algún tipo de inestabilidad para el paciente. Por ejemplo, en las infecciones tardías cuando ya ha ocurrido un proceso de osificación sólida ${ }^{(80,87,93,94)}$.

Asimismo, diferir el cierre de la herida y la instauración de un sistema al vacío (VAC) puede facilitar los lavados posteriores, ya que estos pacientes deben tener un seguimiento estricto, dado el elevado riesgo de

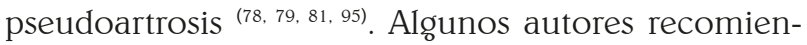
dan el retiro definitivo del instrumental en los casos de infección profunda en los que ya es evidente un compromiso óseo marcado (osteomielitis vertebral y/o absceso intervertebral), ya que de una u otra forma hay pérdida de la estabilidad por el aflojamiento del material y preservarlo conllevaría a prolongar el tiempo de infección, a un mayor número de intervenciones adicionales fallidas y a una mayor destrucción ósea con pseudoartrosis final ${ }^{(78,79,81,82,85)}$.

También, existen estudios a favor del uso de cemento de metilmetacrilato impregnado de antibiótico, especialmente cuando los defectos de cubrimiento son marcados debido al retiro de tejido desvitalizado ${ }^{(82,85,96)}$. Posterior a la finalización del antibiótico endovenoso intrahospitalario (por 15 días), puede ser útil la continuación enteral con el espectro antimicrobiano correspondiente, hasta que se identifique un foco de osificación consolidado y sea pertinente el retiro del material de osteosíntesis ${ }^{(76,84,85,91,93)}$.

\section{MENINGITIS Y VENTRICULITIS POSOPERATORIA O ASOCIADA A DISPOSITIVOS MÉDICOS}

Para la sospecha diagnóstica se deben tener en cuenta signos y síntomas sugestivos, entre los que destacan: la cefalea de novo, náuseas, los cambios en el estado mental o letarogia, siognos de irritación meníngea, eritema o la hipersensibilidad sobre el área donde se implantó el dispositivo, fiebre en ausencia de otro foco infeccioso evidente, peritonitis o pleuritis en pacientes con derivaciones ventriculoperitoneales y ventriculopleurales, respectivamente, y la presencia de bacteriemia sin otra causa que la justifique ${ }^{(12,97-100)}$. EI análisis de LCR puede no proveer la información acerca de esta entidad, debido a que los cambios en la celularidad, glucorraquia y proteinorraquia no son indicadores sensibles de la presencia de infección en el escenario posquirúrgico, y un análisis normal tampoco excluye la infección ${ }^{(101,102)}$.

Debido a lo anterior, los cultivos de LCR son las pruebas más importantes para establecer el diagnóstico, con aumento de su sensibilidad en la presencia de 
hipoglucorraquia $y / 0$ pleocitosis, sin embargo, pueden estar negativos la mayoría de las veces ${ }^{(12,98,99,102)}$. Por esto, los reactantes de la fase aguda como la procalcitonina, el lactato y la proteína $C$ reactiva en LCR pueden ser útiles en el diagnóstico. Así es, por ejemplo, que la literatura reporta valores aceptables de sensibilidad, especificidad y área bajo la curva para el lactato en los niveles que oscilan entre $3,45 \mathrm{mmol} / \mathrm{L}$ a $5,4 \mathrm{mmol} / \mathrm{L}$ $(4,41 \pm 0,85 \mathrm{mmol} / \mathrm{L})^{(103)}$. Igualmente, el $\beta$-oglucano y galactomanano son marcadores útiles para el diagnóstico de la meningitis y ventriculitis fúngica ${ }^{(7)}$.

La terapia antimicrobiana empírica se basa en la administración de agentes que puedan penetrar el SNC y lograr adecuadas concentraciones en LCR, como la vancomicina y un betalactámico con actividad antipseudomonas como el cefepime, ceftazidima o meropenem, el cual debe ser elegido de acuerdo al perfil de sensibilidad del microoronanismo y a las características propias del paciente ${ }^{(104)}$.

La terapia empírica debe iniciarse cuando se sospeche de una infección en presencia de pleocitosis en LCR y posterior a la toma de cultivos, sin esperar el resultado del mismo ${ }^{(104)}$. El tratamiento dirigido deberá realizarse de acuerdo al aislamiento microbiológico y a su patrón de resistencia, con énfasis en el SAMR, en el cual la primera línea es la vancomicina; aunque se han considerado otras estrategias en caso de CIM mayor o igual a $1^{(57,96,105-114)}$ (Tabla 1 y 2). La duración del tratamiento se encuentra en un rango de 4 a 47 días con un promedio de 17,4 días; sin embargo, el tiempo de terapia no parece asociarse a la incidencia de reinfección ${ }^{(15)}$.

Debe considerarse dentro del tratamiento el retiro de catéteres o dispositivos en pacientes con derivación o drenaje de LCR. Anteriormente, se realizaba la administración de antimicrobianos intraventriculares para evitar la morbilidad de los procedimientos adicionales; sin embargo, la tasa de éxito era baja (34-36 \%) y presentaba una mayor mortalidad, en parte por la capacidad de algunos microorganismos de generar biopelícula (biofilm), lo que reducía el éxito de la terapia antimicrobiana y llevaba a una tasa de recurrencia del $70 \%$ si no se retiraba el dispositivo ${ }^{(115,116-118)}$

La práctica más común considera la ađministración de antimicrobianos sistémicos más la remoción de los dispositivos, seguido de la inserción de un sistema de drenaje ventricular, lo que aumenta la tasa de éxito y disminuye las complicaciones ${ }^{(112,115-119)}$. En estas prácticas mencionadas, una vez los cultivos del drenaje ventricular son negativos, se remueve el drenaje y se insertan nuevamente los dispositivos de 2 a 10 días después, de acuerdo al microorganismo aislado ${ }^{(116,119,120)}$.

\section{ANTIBIÓTICOS INTRATECALES/ INTRAVENTRICULARES}

La terapia intratecal/intraventricular consiste en la administración directa, en el flujo de líquido cefalorraquídea, de antibióticos activos contra infecciones en el SNC ${ }^{(120-125)}$. Las ventajas de esta terapia radican en conseguir mayores concentraciones de antimicrobianos en el SNC, que de otra forma no sería posible por las barreras anatómicas y microscópicas propias de este sistema, asociado a una menor circulación sistémica del fármaco ${ }^{(121-125)}$.

No obstante, esta terapia no está aprobada por la Food Drug Administration (FDA), por lo cual su uso está considerado fuera de indicación (off-label). Esto sumado a la carencia de evidencia para su uso de primera línea, limita esta terapia a casos concretos en los cuales no se ha obtenido una respuesta satisfactoria (clínica y paraclínica) a la terapia sistémica de acuerdo al criterio médico (121-125). Además, de acuerdo a la evidencia disponible, esta terapia está contraindicada en neonatos, dado que una revisión de Cochrane encontró una mayor mortalidad asociada a la administración intratecal/intraventricular de antimicrobianos en esta población ${ }^{(126)}$.

Dentro de las intervenciones de prevención, existe un estudio de casos y controles que usa vancomicina de modo preventivo para la reducción de ISO, cuando se dejan drenajes ventriculares externos con resultados favorables, pero que requieren de estudios prospectivos con mayor poder estadístico ${ }^{(127)}$.

Por otro lado, cuando se decide adoptar esta terapia, debe hacerse a través de dispositivos cerrados, bajo estrictas medidas de asepsia $y$ antisepsia y con posterior clampeo de la sonda por 15-60 minutos para equilibrar las concentraciones del antimicrobiano en el LCR ${ }^{(128-135)}$. De igual manera, para la monitorización de la efectividad de la terapia, se usan elementos clínicos y de laboratorio para el seguimiento del paciente, así como medidas farmacocinéticas tales como la toma de niveles del antibiótico en LCR, las cuales, bajo división por la CIM del microorganismo aislado, debe arrojar constantes entre 10 y $20^{(128-135)}$. 
Con respecto a la elección, las dosis y los intervalos de la administración de antimicrobianos, las decisiones deben tener en consideración el microorganismo aislado y su patrón de resistencia, la neurotoxicidad del agente y la salida diaria de LCR por el drenaje externo ${ }^{(109,128-135,136-148)}$ (Tabla 3 y Tabla 4).

Tabla 3. Listado de antimicrobianos para el uso sistémico

\begin{tabular}{|c|c|c|}
\hline Antimicrobiano & Dosis SNC & Dosis usual * \\
\hline Amikacina & $\begin{array}{l}\text { Niños: } 20-30 \text { mg/kg (en } 3 \text { dosis) } \\
\text { Adultos: } 15 \text { mg/kg (en } 3 \text { dosis) }\end{array}$ & $\begin{array}{l}\text { Niños: } 15 \text { - 22,5 mg/kg (en } 3 \text { dosis) } \\
\text { Adultos: } 15 \text { mg/kg (en } 3 \text { dosis) }\end{array}$ \\
\hline Ampicilina & $\begin{array}{l}\text { Niños: } 300-400 \mathrm{mg} / \mathrm{kg} \text { (en } 4 \text { dosis) } \\
\text { Adultos: } 12 \mathrm{~g} \text { (en } 4 \text { dosis) }\end{array}$ & $\begin{array}{l}\text { Niños: } 100 \text { - } 150 \text { mg/kg (en } 4 \text { dosis) } \\
\text { Adultos: } 12 \text { g e (en infusión continua o en } 6 \text { dosis) }\end{array}$ \\
\hline Aztreonam & $\begin{array}{l}\text { Niños: } 120 \text { mg/kg (en 3-4 dosis) } \\
\text { Adultos: 6-8 g (En 3-4 dosis) }\end{array}$ & $\begin{array}{l}\text { Niños: } 90 \text { mg/kg (en } 3 \text { dosis) } \\
\text { Adultos: } 6 \mathrm{~g} \text { (en } 3 \text { dosis) }\end{array}$ \\
\hline Cefepime & $\begin{array}{l}\text { Niños: } 150 \text { mg/kg (en } 3 \text { dosis) } \\
\text { Adultos: } 6 \mathrm{~g} \text { (en } 3 \text { dosis) }\end{array}$ & $\begin{array}{l}\text { Niños: } 100 \text { mg/kg (en } 2 \text { dosis) } \\
\text { Adultos } 6 \mathrm{~g} \text { (en } 3 \text { dosis) }\end{array}$ \\
\hline Cefotaxime & $\begin{array}{l}\text { Niños: } 300 \text { mg/kg (En 3-4 dosis) } \\
\text { Adultos: 8-12 g (En 4-6 dosis) }\end{array}$ & $\begin{array}{l}\text { Niños: } 50 \text { - } 180 \text { mg/kg (en 3-4 dosis) } \\
\text { Adultos: } 3 \text { - } 6 \text { g (en } 3 \text { dosis) }\end{array}$ \\
\hline Ceftriaxona & $\begin{array}{l}\text { Niños: } 100 \text { mg/kg (en 1-2 dosis) } \\
\text { Adultos: } 4 \text { g (en } 2 \text { dosis) }\end{array}$ & $\begin{array}{l}\text { Niños: } 50-75 \text { mg/kg (en } 1 \text { dosis) } \\
\text { Adultos: } 2 \mathrm{~g} \text { (en } 1 \text { dosis) }\end{array}$ \\
\hline Ceftazidime & $\begin{array}{l}\text { Niños: } 200 \text { mg/kg (en } 3 \text { dosis) } \\
\text { Adultos: } 6 \text { g (en } 3 \text { dosis) }\end{array}$ & $\begin{array}{l}\text { Niños: } 90-150 \text { mg/kg (en } 3 \text { dosis) } \\
\text { Adultos: } 6 \mathrm{~g} \text { (en } 3 \text { dosis) }\end{array}$ \\
\hline Ciprofloxacina & $\begin{array}{l}\text { Niños: } 30 \text { mg/kg (en 2-3 dosis) } \\
\text { Adultos: } 800-1200 \text { mg (en 2-3 dosis) }\end{array}$ & $\begin{array}{l}\text { Niños: } 20 \text { mg/kg (en } 2 \text { dosis) } \\
\text { Adultos: } 400 \text { mg IV cada } 8 \text { horas }\end{array}$ \\
\hline Daptomicina & $\begin{array}{l}\text { Sin información } \\
\text { Adultos: 6-10 mg/kg (en } 1 \text { dosis) }\end{array}$ & $\begin{array}{l}\text { Niños: } 6-10 \text { mg/kg (en } 1 \text { dosis) } \\
\text { Adultos: } 6-8 \text { mg/kg (en } 1 \text { dosis) }\end{array}$ \\
\hline Gentamicina & $\begin{array}{l}\text { Niños: } 75 \text { mg/kg (en } 3 \text { dosis) } \\
\text { Adultos: } 5 \text { mg/kg (en } 3 \text { dosis) }\end{array}$ & $\begin{array}{l}\text { Niños: } 6-7,5 \text { mg/kg (en } 3 \text { dosis) } \\
\text { Adultos: } 3-5 \text { mg/kg (en } 3 \text { dosis) }\end{array}$ \\
\hline Linezolid & $\begin{array}{l}\text { Niños: < } 12 \text { a } 30 \text { mg/kg (en } 3 \text { dosis) } \\
\text { > } 12 \text { a } 20 \text { mg/kg (en } 2 \text { dosis) } \\
\text { Adultos: } 1200 \text { mg (en } 2 \text { dosis) }\end{array}$ & $\begin{array}{l}\text { Niños: } 30 \text { mg/kg (en } 3 \text { dosis) } \\
\text { Adultos: } 1200 \text { mg (en } 2 \text { dosis) }\end{array}$ \\
\hline Meropenem & $\begin{array}{l}\text { Niños: } 120 \text { mg/kg (en } 3 \text { dosis) } \\
\text { Adultos: } 6 \mathrm{~g} \text { (en } 3 \text { dosis) }\end{array}$ & $\begin{array}{l}\text { Niños: } 60 \text { mg/kg (en } 3 \text { dosis) } \\
\text { Adultos: } 3 \mathrm{~g} \text { (en } 3 \text { dosis) }\end{array}$ \\
\hline Moxifloxacino & $\begin{array}{l}\text { No recomendado } \\
\text { Adultos: } 400 \text { mg (en } 1 \text { dosis) }\end{array}$ & $\begin{array}{l}\text { Niños: No recomendado } \\
\text { Adultos: } 400 \text { mg (en } 1 \text { dosis) }\end{array}$ \\
\hline Oxacilina & $\begin{array}{l}\text { Niños: } 200 \text { mg/kg (en } 4 \text { dosis) } \\
\text { Adultos: } 12 \mathrm{~g} \text { (en } 6 \text { dosis) }\end{array}$ & $\begin{array}{l}\text { Niños: } 100-150 \mathrm{mg} / \mathrm{kg} \text { (en } 4 \text { dosis) } \\
\text { Adultos: } 12 \mathrm{~g} \text { (en } 6 \text { dosis) o infusión continua }\end{array}$ \\
\hline Penicilina G & $\begin{array}{l}\text { Niños: } 300000 \mathrm{UI} / \mathrm{kg} \text { (en } 4-6 \text { dosis) } \\
\text { Adultos: } 24 \text { millones UI (en } 6 \text { dosis) }\end{array}$ & $\begin{array}{l}\text { Niños: } 100000-150000 \mathrm{UI} / \mathrm{kg} \text { (en } 4 \text { dosis) } \\
\text { Adultos: } 20-24 \text { millones UI (en infusión continua o en } 6 \text { dosis) }\end{array}$ \\
\hline Rifampicina & $\begin{array}{l}\text { Niños: } 20 \text { mg/kg (en } 1 \text { dosis) } \\
\text { Adultos: } 600 \text { mg (en } 1 \text { dosis) }\end{array}$ & $\begin{array}{l}\text { Niños: } 20 \text { mg/kg (en } 1 \text { o } 2 \text { dosis) } \\
\text { Adultos: } 600 \text { mg (en } 1 \text { dosis) }\end{array}$ \\
\hline Trimetoprim-Sulfametoxazol & $\begin{array}{l}\text { Niños: } 10-20 \mathrm{mg} / \mathrm{kg} \text { (en } 2-4 \text { dosis) } \\
\text { Adultos: } 10-20 \mathrm{mg} / \mathrm{kg} \text { (en } 2-4 \text { dosis) }\end{array}$ & $\begin{array}{l}\text { Niños: } 16-24 \mathrm{mg} / \mathrm{kg} \mathrm{kg} \text { (en } 2 \text { dosis) } \\
\text { Adultos: } 8 \mathrm{mg} / \mathrm{kg} \text { (en } 2 \text { dosis) }\end{array}$ \\
\hline Vancomicina & $\begin{array}{l}\text { Niños: } 60 \mathrm{mg} / \mathrm{kg} \text { (en } 4 \text { dosis) } \\
\text { Adultos: } 30-60 \mathrm{mg} / \mathrm{kg} \text { (en } 2-3 \text { dosis) }\end{array}$ & $\begin{array}{l}\text { Niños: } 40 \text { mg/kg (en } 4 \text { dosis) } \\
\text { Adultos: } 30-40 \text { mg/kg (en } 2 \text { dosis) }\end{array}$ \\
\hline Fluconazol & $\begin{array}{l}\text { Candidiasis } \\
\text { Niños: } 6-12 \mathrm{mg} / \mathrm{kg} \text { (en 1-2 dosis) } \\
\text { Adultos: } 400-800 \mathrm{mg} \text { (en 1-2 dosis) }\end{array}$ & $\begin{array}{l}\text { Candidiasis } \\
\text { Niños: } 6-12 \text { mg/kg (en } 1 \text { dosis) } \\
\text { Adultos: } 400 \text { mg (en } 1 \text { dosis) }\end{array}$ \\
\hline
\end{tabular}


Tabla 3. Listado de antimicrobianos para el uso sistémico (Continuación)

\begin{tabular}{lll}
\hline Anfotericina B (Liposomal) & Niños: $6 \mathrm{mg} / \mathrm{kg}$ (en 1 dosis) & Niños: $6 \mathrm{mg} / \mathrm{kg}$ (en 1 dosis) \\
Aspergillus & Adultos: $6 \mathrm{mg} / \mathrm{kg}$ (en 2 dosis) & Adultos: $6 \mathrm{mg} / \mathrm{kg}$ (en 1 dosis) \\
$\begin{array}{lll}\text { Anfotericina B (Liposomal) } \\
\text { Cándida }\end{array}$ & Niños: $5 \mathrm{mg} / \mathrm{kg}$ (en 1 dosis) & Niños: $3-5 \mathrm{mg} / \mathrm{kg}$ (en 1 dosis) \\
\hline
\end{tabular}

SNC: sistema nervioso central. * Dosis empleada en la infección del sitio operatorio superficial y profunda, y en la osteomielitis vertebral sin el compromiso de las estructuras del sistema nervioso central. Fuente: Creación propia

Tabla 4. Listado de antimicrobianos para el uso intratecal/intraventricular

\begin{tabular}{|c|c|}
\hline Dosis & RAM severas \\
\hline \multicolumn{2}{|c|}{ Vancomicina } \\
\hline Dosis general: $10-20$ mg cada 24 horas & Pérdida temporal de la audición \\
\hline \multicolumn{2}{|l|}{ Dosis de acuerdo con el tamaño de los ventrículos: } \\
\hline \multicolumn{2}{|l|}{ Pequeños: 5 mg } \\
\hline \multicolumn{2}{|l|}{ Normal: $10 \mathrm{mg}$} \\
\hline \multicolumn{2}{|l|}{ Ventriculomegalia: $15-20 \mathrm{mg}$} \\
\hline \multicolumn{2}{|c|}{ Dosis e intervalos de administración de acuerdo con el volumen de drenaje externo: } \\
\hline \multicolumn{2}{|l|}{$<50 \mathrm{ml} /$ día: cada 72 horas } \\
\hline \multicolumn{2}{|l|}{ 50-100 ml/día: cada 48 horas } \\
\hline \multicolumn{2}{|l|}{ 100-150 ml/día: cada 24 horas } \\
\hline \multicolumn{2}{|l|}{ 150-200 ml/día: aumentar la dosis 5 mg; intervalo cada 24 horas } \\
\hline 200-250 ml/día: aumentar la dosis 10 mg; intervalo cada 24 horas & \\
\hline
\end{tabular}

Daptomicina

5-10 mg cada 24-72 horas

Gentamicina

Dosis general: 4-10 mg cada 24 horas

Dosis de acuerdo con el tamaño de los ventrículos:

Pequeños: $2 \mathrm{mg}$

Normal: $3 \mathrm{mg}$

Ventriculomegalia: $4-5 \mathrm{mg}$

Dosis e intervalos de administración de acuerdo con el volumen del drenaje externo:

$<50 \mathrm{ml} /$ día: cada 72 horas

$50-100 \mathrm{ml} /$ día: cada 48 horas

100-150 ml/día: cada 24 horas

150-200 ml/día: aumentar la dosis $5 \mathrm{mg}$; intervalo cada 24 horas

200-250 ml/día: aumentar la dosis 10 mg; intervalo cada 24 horas

\section{Amikacina}

$30 \mathrm{mg}$ (5-50 mg) cada 24 horas

Meropenem

10 mg cada 12 horas

\section{Fiebre}

Pérdida temporal de la audición.

Convulsiones.

Meningitis aséptica.

Pleocitosis eosinofílica en LCR. 
Tabla 4. Listado de antimicrobianos para el uso intratecal/intraventricular (Continuación)

\begin{tabular}{|c|c|}
\hline \multicolumn{2}{|c|}{ Polimixinas } \\
\hline $\begin{array}{l}\text { Polimixina E (colistina): } 10 \text { mg cada } 24 \text { horas } \\
\text { Polimixina B: } 5 \text { mg cada } 24 \text { horas }\end{array}$ & $\begin{array}{l}\text { Inflamación meníngea } \\
\text { Convulsiones con altas dosis. } \\
\text { Pérdida del apetito } \\
\text { Agitación } \\
\text { Eosinofilia, edema, dolor. } \\
\text { Albuminuria } \\
\text { Síndrome parecido a la miastenia gravis }\end{array}$ \\
\hline \multicolumn{2}{|c|}{ Tigeciclina } \\
\hline $\begin{array}{l}1 \mathrm{mg} \text { cada } 12 \text { horas }+49 \mathrm{mg} \text { IV cada } 12 \text { horas ó } \\
5 \mathrm{mg} \text { cada } 12 \text { horas }+45 \mathrm{mg} \text { IV cada } 12 \text { horas ó } \\
10 \mathrm{mg} \text { cada } 12 \text { horas y } 40 \mathrm{mg} \text { IV cada } 12 \text { horas }\end{array}$ & No reportados \\
\hline \multicolumn{2}{|c|}{ Quinupristina/dalfopristina } \\
\hline $2-5 \mathrm{mg}$ & No es claro, 1 reporte de caso. \\
\hline \multicolumn{2}{|c|}{ Tobramicina } \\
\hline $5-20 \mathrm{mg}$ & Similar a la gentamicina \\
\hline \multicolumn{2}{|c|}{ Anfotericina B } \\
\hline $0,1-0,5$ mg cada 24 horas en DAD $5 \%$ & Tinnitus, fiebre, parkinsonismo, escalofrío. \\
\hline
\end{tabular}

RAM: Reacción adversa al medicamento. IV: intravenoso. LCR: líquido cefalorraquídeo. DAD: dextrosa en agua destilada. Fuente: creación propia

\section{DISCUSIÓN}

Las ISO en neurocirugía son una amenaza para el éxito de las intervenciones quirúrgicas y se asocian con una alta carga de morbilidad, mortalidad y $\operatorname{costos}^{(1-4)}$. Esto asociado a la problemática de multirresistencia a antimicrobianos, requiere que las instituciones y el personal de salud conozcan las intervenciones con un mejor costo de acuerdo a su efectividad para la prevención y el tratamiento de las ISO ${ }^{(5,149)}$. Enunciadas ya dichas intervenciones, es importante establecer principios éticos en el desarrollo de estudios clínicos donde se evalúen intervenciones para la prevención y tratamiento de las ISO, que tengan en cuenta el beneficio, los costos, las reacciones adversas y el posible desarrollo de resistencias bacterianas ${ }^{(149)}$. De igual forma, los profesionales de la salud deberán adherirse a protocolos de atención que proporcionen el mayor beneficio a sus pacientes ${ }^{(149)}$.

Desde esta perspectiva, los estudios de costo-efectividad son relevantes, tal como se ha realizado con los catéteres impregnados de antibiótico o con el uso tópico de vancomicina. Asimismo, se requieren estudios de seguridad in vivo e in vitro ${ }^{(65,149-153)}$. De este modo, muchas decisiones se tomarán en relación con el riesgo y el beneficio para el paciente, en el contexto de un asunto que está en continuo desarrollo y en el que existen varios cuestionamientos que requieren mayor investigación para tomar decisiones clínicas basadas en la evidencia. Algunos de esos temas por investigar se relacionan con establecer la efectividad y la seguridad de los protocolos de atención, del uso sistémico y tópico de antibióticos profilácticos con esquemas definidos, de la orientación más objetiva sobre el retiro de material de osteosíntesis, prótesis y de dispositivos médicos intracraneales y de la terapia antimicrobiana intratecal/ intraventricular. Finalmente, se debe recalcar en la necesidad de la publicación de más estudios farmacocinéticos que puedan guiar de manera más exacta la terapia antimicrobiana en las infecciones del SNC, con enfoque en medicina personalizada y de precisión ${ }^{(154)}$.

\section{CONCLUSIONES}

Las ISO en neurocirugía son eventos frecuentes, clínicamente relevantes, con implicaciones importantes 
en morbilidad y mortalidad para los pacientes y en los costos para el sistema de salud. Es por esta razón, que se requiere de la actualización sintética del conocimiento médico sobre la prevención y el tratamiento de este tipo de infecciones.

\section{CONFLICTOS DE INTERESES}

Ninguno por declarar.

\section{REFERENCIAS BIBLIOGRÁFICAS}

1. Herwaldt LA, Cullen JJ, Scholz D, French P, Zimmerman MB, Pfaller MA, et al. A prospective study of outcomes, healthcare resource utilization, and costs associated with postoperative nosocomial infections. Infect Control Hosp Epidemiol. 2006;27(12):1291-8. DOI 10.1086/509827.

2. McGirt MJ, Godil SS. Reduction of surgical site infection in spine surgery: an opportunity for quality improvement and cost reduction. Spine J. 2013;13(9):1030-1. DOI 10.1016/j.spinee.2013.06.003.

3. O'Keeffe AB, Lawrence T, Bojanic S. Oxford craniotomy infections database: a cost analysis of craniotomy infection. Br J Neurosurg. 2012;26(2):265-9. DOI 10.3109/02688697.2011.626878.

4. Saavedra CH, Ordonez KM, Diaz JA. [Nosocomial infections impact in a hospital in Bogota, Colombia: effects on mortality and hospital costs]. Rev Chilena Infectol. 2015;32(1):25-9. DOI 10.4067/S071610182015000200006 .

5. Barlam TF, Cosgrove SE, Abbo LM, MacDougall C, Schuetz AN, Septimus EJ, et al. Implementing an Antibiotic Stewardship Pro spp spp : Guidelines by the Infectious Diseases Society of America and the Society for Healthcare Epidemiology of America. Clin Infect Dis. 2016;62(10):e51-77. DOI 10.1093/cid/ciw 118.

6. Fajardo Rodríguez HA, Quemba Gordillo J, Eslava Schmalbach J. [Prediction scales and infection on surgical sites in 15625 surgeries. 2001-2003]. Rev Salud Publica (Bogota). 2005;7(1):89-98.

7. Tunkel AR, Hasbun R, Bhimraj A, Byers K, Kaplan SL, Michael Scheld W, et al. 2017 Infectious Diseases Society of America's Clinical Practice Guidelines for Healthcare-Associated Ventriculitis and Meningitis. Clin Infect Dis. 2017. DOI 10.1093/cid/ciw861.
8. Alvarez-Moreno C, Perez-Fernandez AM, Rosenthal VD, Quintero J, Chapeta-Parada E, Linares C, et aI. Surgical site infection rates in 4 cities in Colombia: findings of the International Nosocomial Infection Control Consortium (INICC). Am J Infect Control. 2014;42(10):1089-92. DOI 10.1016/j.ajic.2014.06.010.

9. Edwards JR, Peterson KD, Mu Y, Banerjee S, Allen-Bridson K, Morrell G, et al. National Healthcare Safety Network (NHSN) report: data summary for 2006 through 2008, issued December 2009. Am J Infect Control. 2009;37(10):783-805. DOI 10.1016/j.ajic.2009.10.001.

10. Gerometta A, Rodriguez Olaverri JC, Bitan F. Infections in spinal instrumentation. International orthopaedics. Int Orthop. 2012;36(2):457-64. DOI 10.1007/ s00264-011-1426-0.

11. Governale LS, Fein N, Logsdon J, Black PM. Techniques and complications of external lumbar drainage for normal pressure hydrocephalus. Neurosurgery. 2008;63(4 SuppI 2):379-84;379-84. DOI 10.1227/01. NEU.0000327023.18220.88.

12. Lozier AP, Sciacca RR, Romagnoli MF, Connolly ES. Ventriculostomy-related Infections: A Critical Review of the Literature. Neurosurgery. 2002;51(1):170-82.

13. McClelland S, 3rd. Postoperative intracranial neurosurgery infection rates in North America versus Europe: a systematic analysis. Am J Infect Control. 2008;36(8):570-3. DOI 10.1016/j.ajic.2007.07.015.

14. Radcliff KE, Neusner AD, Millhouse PW, Harrop JD, Kepler CK, Rasouli MR, et al. What is new in the diagnosis and prevention of spine surgical site infections. Spine J. 2015;15(2):336-47. DOI 10.1016/j. spinee.2014.09.022.

15. Ramanan M, Lipman J, Shorr A, Shankar A. A metaanalysis of ventriculostomy-associated cerebrospinal fluid infections. BMC Infect Dis. 2015;15:3. DOI 10.1186/s12879-014-0712-z.

16. Rosenthal VD, Richtmann R, Singh S, Apisarnthanarak A, Kubler A, Viet-Hung N, et al. Surgical site infections, International Nosocomial Infection Control Consortium (INICC) report, data summary of 30 countries, 2005-2010. Infect Control Hosp Epidemiol. 2013;34(6):597-604. DOI 10.1086/670626.

17. Kourbeti IS, Vakis AF, Ziakas P, Karabetsos D, Potolidis E, Christou S, et al. Infections in patients undergoing craniotomy: risk factors associated with post-craniotomy meningitis. J Neurosurg. 2015;122(5):1113-9. DOI 10.3171/2014.8.JNS132557. 
18. Ploegmakers IB, OIde Damink SW, Breukink SO. AIternatives to antibiotics for prevention of surgical infection. Br J Surg. 2017;104(2):e24-e33. DOI 10.1002/ bjs. 10426 .

19. Seicean A, Kumar P, Seicean S, Neuhauser D, SeIman WR, Bambakidis NC. Impact of Resident Involvement in Neurosurgery: An American College of Surgeons' National Surgical Quality Improvement Program Database Analysis of 33,977 Patients. Neurospine. 2018;15(1):54-65. DOI 10.14245/ns.1836008.004.

20. Sarmey N, Kshettry VR, Shriver MF, Habboub G, Machado AG, Weil RJ. Evidence-based interventions to reduce shunt infections: a systematic review. Childs Nerv Syst. 2015;31(4):541-9. DOI 10.1007/s00381-015-2637-2.

21. Hill M, Baker G, Carter D, Henman LJ, Marshall K, Mohn K, et al. A multidisciplinary approach to end external ventricular drain infections in the neurocritical care unit. J Neurosci Nurs. 2012;44(4):188-93. DOI 10.1097/JNN.obo13e3182527672.

22. Kestle JR, Riva-Cambrin J, Wellons JC, 3rd, Kulkarni AV, Whitehead WE, Walker ML, et al. A standardized protocol to reduce cerebrospinal fluid shunt infection: the Hydrocephalus Clinical Research Network Quality Improvement Initiative. J Neurosurg Pediatr. 2011;8(1):22-9. DOI 10.3171/2011.4.PEDS10551.

23. Rivero-Garvia M, Marquez-Rivas J, Jimenez-Mejias ME, Neth O, Rueda-Torres AB. Reduction in external ventricular drain infection rate. Impact of a minimal handling protocol and antibiotic-impregnated catheters. Acta Neurochir (Wien). 2011;153(3):64751. DOI 10.1007/s00701-010-0905-1.

24. Rozzelle CJ, Leonardo J, Li V. Antimicrobial suture wound closure for cerebrospinal fluid shunt surgery: a prospective, double-blinded, randomized controlled trial. J Neurosurg Pediatr. 2008;2(2):111-7. DOI 10.3171/PED/2008/2/8/111.

25. Govender ST, Nathoo N, van Dellen JR. Evaluation of an antibiotic-impregnated shunt system for the treatment of hydrocephalus. J Neurosurg. 2003;99(5):831-9. DOI 10.3171/jns.2003.99.5.0831.

26. Harrop JS, Sharan AD, Ratliff J, Prasad S, Jabbour P, Evans JJ, et al. Impact of a standardized protocol and antibiotic-impregnated catheters on ventriculostomy infection rates in cerebrovascular patients. Neurosurgery. 2010;67(1):187-91. DOI 10.1227/01. NEU.0000370247.11479.B6.
27. Konstantelias AA, Vardakas KZ, Polyzos KA, TansarIi GS, Falagas ME. Antimicrobial-impregnated and -coated shunt catheters for prevention of infections in patients with hydrocephalus: a systematic review and meta-analysis. J Neurosurg. 2015;122(5):1096112. DOI 10.3171/2014.12.JNS14908.

28. Parker SL, Anderson WN, Lilienfeld S, Megerian JT, McGirt MJ. Cerebrospinal shunt infection in patients receiving antibiotic-impregnated versus standard shunts. Journal of neurosurgery Pediatrics. 2011;8(3):259-65.

29. Parker SL, McGirt MJ, Murphy JA, Megerian JT, Stout M, Engelhart L. Comparative effectiveness of antibioticimpregnated shunt catheters in the treatment of adult and pediatric hydrocephalus: analysis of 12,589 consecutive cases from 287 US hospital systems. J Neurosurg. 2015;122(2):443-8. DOI 10.3171/2014.10.JNS13395.

30. Root BK, Barrena BG, Mackenzie TA, Bauer DF. Antibiotic Impregnated External Ventricular Drains: Meta and Cost Analysis. World Neurosurg. 2016;86:306-15. DOI 10.1016/j.wneu.2015.09.032.

31. Sonabend AM, Korenfeld Y, Crisman C, Badjatia N, Mayer SA, Connolly ES Jr. Prevention of ventriculostomy-related infections with prophylactic antibiotics and antibiotic-coated external ventricular drains: a systematic review. Neurosurgery. 2011;68(4):9961005. DOI 10.1227/NEU.ob013e3182096d84.

32. Tamburrini G, Massimi L, Caldarelli M, Di Rocco C. Antibiotic impregnated external ventricular drainage and third ventriculostomy in the management of hydrocephalus associated with posterior cranial fossa tumours. Acta Neurochir (Wien). 2008;150(10):104955. DOI 10.1007/s00701-008-0022-6.

33. Thomas R, Lee S, Patole S, Rao S. Antibiotic-impregnated catheters for the prevention of CSF shunt infections: a systematic review and metaanalysis. Br J Neurosurg. 2012;26(2):175-84. DOI 10.3109/02688697.2011.603856.

34. Kan P, Kestle J. Lack of efficacy of antibiotic-impregnated shunt systems in preventing shunt infections in children. Childs Nerv Syst. 2007;23(7):773-7. DOI 10.1007/s00381-007-0296-7.

35. Ritz R, Roser F, Morgalla M, Dietz K, Tatagiba M, Will BE. Do antibiotic-impregnated shunts in hydrocephalus therapy reduce the risk of infection? An observational study in 258 patients. BMC Infect Dis. 2007;7:38. DOI 10.1186/1471-2334-7-38. 
36. McLaughlin N, St-Antoine P, Bojanowski MW. Impact of antibiotic-impregnated catheters on the timing of cerebrospinal fluid infections in non-traumatic subarachnoid hemorrhage. Acta Neurochir (Wien). 2012;154(4):761-7. DOI 10.1007/s00701-012-1276-6.

37. Sciubba DM, McGirt MJ, Woodworth GF, Carson B, Jallo GI. Prolonged exposure to antibiotic-impregnated shunt catheters does not increase incidence of late shunt infections. Childs Nerv Syst. 2007;23(8):867-71. DOI 10.1007/s00381-007-0334-5.

38. Wong GKC, Poon WS, Stephanie CPN, Ip M. The impact of ventricular catheter impregnated with antimicrobial agents on infections in patients with ventricular catheter: interim report. Acta Neurochir Suppl. 2008;102:53-5. DOI 10.1007/978-3-211-85578-2_11.

39. Murphy RK, Liu B, Srinath A, Reynolds MR, Liu J, Craighead $M C$, et al. No additional protection against ventriculitis with prolonged systemic antibiotic prophylaxis for patients treated with antibioticcoated external ventricular drains. J Neurosurg. 2015;122(5):1120-6. DOI 10.3171/2014.9.JNS132882.

40. Cui Z, Wang B, Zhong Z, Sun Y, Sun Q, Yang G, et al. Impact of antibiotic- and silver-impregnated external ventricular drains on the risk of infections: A systematic review and meta-analysis. Am J Infect Control. 2015;43(7):e23-32. DOI 10.1016/j.ajic.2015.03.015.

41. Burrows AM, Murphy ME, DanieIs DJ, Meyer FB. Antibiotic Reservoir Injection Reduces Shunt Infection in Adults. World Neurosurg. 2016;89:108-11. DOI 10.1016/j.wneu.2016.01.077.

42. Ragel BT, Browd SR, Schmidt RH. Surgical shunt infection: significant reduction when using intraventricular and systemic antibiotic agents. J Neurosurg. 2006; 105(2):242-7. DOI 10.3171/jns.2006.105.2.242.

43. Leib SL, Boscacci R, Gratzl O, Zimmerli W. Predictive value of cerebrospinal fluid (CSF) lactate level versus CSF/blood glucose ratio for the diagnosis of bacterial meningitis following neurosurgery. Clin Infect Dis. 1999;29(1):69-74. DOI 10.1086/520184.

44. Abraham P, Lamba N, Acosta M, Gholmie J, Dawood HY, Vestal $M$, et al. Antibacterial prophylaxis for gram-positive and gram-negative infections in cranial surgery: A meta-analysis. J Clin Neurosci. 2017;45:24-32. DOI 10.1016/j.jocn.2017.07.039.

45. Alotaibi AF, Hulou MM, Vestal M, Alkholifi F, Asgarzadeh $\mathrm{M}$, Cote DJ, et al. The Efficacy of Antibacterial Prophylaxis Against the Development of Meningitis
After Craniotomy: A Meta-Analysis. World Neurosurg. 2016;90:597-603 e1. DOI 10.1016/j.wneu.2016.02.048.

46. Haines S, Walters B. Antibiotic prophylaxis for cerebrospinal fluid shunts: a metanalysis. Neurosurgery. 1994;34(1):87-92.

47. Klimo P, Jr., Van Poppel M, Thompson CJ, Baird LC, Duhaime AC, Flannery AM, et al. Pediatric hydrocephalus: systematic literature review and evidence-based guidelines. Part 6: Preoperative antibiotics for shunt surgery in children with hydrocephalus: a systematic review and meta-analysis. J Neurosurg Pediatr. 2014;14(Suppl 1):44-52. DOI 10.3171/2014.7.PEDS14326.

48. Langley JM, LeBlanc JC, Drake J, Milner R. Efficacy of Antimicrobial Prophylaxis in Placement of Cerebrospinal Fluid Shunts: Meta-Analysis. Clin Infect Dis. 1993;17(1):98-103.

49. Ratilal B, Costa J, Sampaio C. Antibiotic prophylaxis for surgical introduction of intracranial ventricular shunts. Cochrane Database Syst Rev. 2006(3):CD005365. DOI 10.1002/14651858.CD005365.pub2.

50. Ratilal B, Costa J, Sampaio C. Antibiotic prophylaxis for surgical introduction of intracranial ventricular shunts: a systematic review. J Neurosurg Pediatr. 2008;1(1):48-56. DOI 10.3171/PED-08/01/048.

51. Xu H, Hu F, Hu H, Sun W, Jiao W, Li R, et al. Antibiotic prophylaxis for shunt surgery of children: a systematic review. Childs Nerv Syst. 2016;32(2):253-8. DOI 10.1007/s00381-015-2937-6.

52. Rosen SA, Getz AE, Kingdom T, Youssef AS, Ramakrishnan VR. Systematic review of the effectiveness of perioperative prophylactic antibiotics for skull base surgeries. Am J Rhinol Allergy. 2016;30(2):e10-6. DOI 10.2500/ajra.2016.30.4298.

53. Tacconelli E, Cataldo MA, Albanese A, Tumbarello M, Arduini E, Spanu T, et al. Vancomycin versus cefazolin prophylaxis for cerebrospinal shunt placement in a hospital with a high prevalence of meticillin-resistant Staphylococcus aureus. J Hosp Infect. 2008;69(4):337-44. DOI 10.1016/j.jhin.2008.04.032.

54. Nejat F, Tajik P, EI Khashab M, Kazmi S, Khotaei G, Salahesh S. A randomized trial of ceftriaxone versus trimethoprim-sulfamethoxazole to prevent ventriculoperitoneal shunt infection. J Microbiol Immunol Infect. 2008;41(2):112-7.

55. Wong GK, Poon WS, Lyon D, Wai S. Cefepime vs. Ampicillin/Sulbactam and Aztreonam as 
antibiotic prophylaxis in neurosurgical patients with external ventricular drain: result of a prospective randomized controlled clinical trial. J Clin Pharm Ther. 2006;31(3):231-5. DOI 10.1111/j.13652710.2006.00729.x.

56. Ghobrial GM, Cadotte DW, Williams K Jr, Fehlings MG, Harrop JS. Complications from the use of intrawound vancomycin in lumbar spinal surgery: a systematic review. Neurosurgical focus. 2015;39(4):E11. DOI 10.3171/2015.7.FOCUS15258.

57. Abdullah KG, Attiah MA, Olsen AS, Richardson A, Lucas TH. Reducing surgical site infections following craniotomy: examination of the use of topical vancomycin. J Neurosurg. 2015;123(6):1600-4. DOI 10.3171/2014.12.JNS142092.

58. Amrani J. Intraoperative powdered vancomycin use with paddle lead placement. Neuromodulation. 2015;18(3):177-80; discussion 181. DOI 10.1111/ ner. 12216 .

59. Bakhsheshian J, Dahdaleh NS, Lam SK, Savage JW, Smith ZA. The use of vancomycin powder in modern spine surgery: systematic review and metaanalysis of the clinical evidence. World Neurosurg. 2015;83(5):816-23. DOI 10.1016/j.wneu.2014.12.033.

60. Hill BW, Emohare O, Song B, Davis R, Kang MM. The use of vancomycin powder reduces surgical reoperation in posterior instrumented and noninstrumented spinal surgery. Acta Neurochir (Wien). 2014;156(4):749-54. DOI 10.1007/s00701-014-2022-z.

61. Should we add vancomycin antibiotic powder to prevent post operative infection in spine surgery? First update. Medwave. 2015;15(Suppl 1):e6160. DOI 10.5867/medwave.2015.6202.

62. López M, Molina M. Should we add vancomycin antibiotic powder to prevent post operative infection in spine surgery? - First update. Medwave. 2015;15 (Suppl 2):e6202.

63. Molinari RW, Khera OA, Molinari WJ 3rd. Prophylactic intraoperative powdered vancomycin and postoperative deep spinal wound infection: 1,512 consecutive surgical cases over a 6-year period. Eur Spine J. 2012;21(Suppl 4):S476-82. DOI 10.1007/s00586-0112104-z.

64. Rasouli JJ, Kopell BH. The Adjunctive Use of Vancomycin Powder Appears Safe and May Reduce the Incidence of Surgical-Site Infections After
Deep Brain Stimulation Surgery. World Neurosurg. 2016;95:9-13. DOI 10.1016/j.wneu.2016.07.063.

65. Ravikumar $\nabla$, Ho AL, Pendhakar AV, Sussman ES, Kwong-Hon Chow K, Li G. The Use of Vancomycin Powder for Surgical Prophylaxis Following Craniotomy. Neurosurgery. 2017;80(5):754-8. DOI 10.1093/ neuros/nyw 127.

66. Strom RG, Pacione D, Kalhorn SP, Frempong-Boadu AK. Lumbar laminectomy and fusion with routine local application of vancomycin powder: decreased infection rate in instrumented and non-instrumented cases. Clin Neurol Neurosurg. 2013;115(9):17669. DOI 10.1016/j.clineuro.2013.04.005.

67. Tomor M, Mitsunaga L, Durbin-Johnson B, NaIlur D, Roberto R. Reducing surgical site infection in spinal surgery with betadine irrigation and intrawound vancomycin powder. Spine (Phila Pa 1976). 2015;40(7):491-9. DOI 10.1097/ BRS.0000000000000789.

68. van Lindert EJ, Bilsen MV, Flier MV, Kolwijck E, Delye $\mathrm{H}$, Oever JT. Topical vancomycin reduces the cerebrospinal fluid shunt infection rate: A retrospective cohort study. PIoS one. 2018;13(1):e0190249. DOI 10.1371/journal.pone.0190249.

69. Tubaki VR, Rajasekaran S, Shetty AP Effects of using intravenous antibiotic only versus local intrawound vancomycin antibiotic powder application in addition to intravenous antibiotics on postoperative infection in spine surgery in 907 patients. Spine (Phila Pa 1976). 2013;38(25):2149-55. DOI 10.1097/ BRS.0000000000000015.

70. Carney N, Totten AM, O'Reilly C, Ullman JS, Hawryluk GW, Bell MJ, et al. Guidelines for the Management of Severe Traumatic Brain Injury, Fourth Edition. Neurosurgery. 2017;80(1):6-15. DOI 10.1227/ NEU.0000000000001432.

71. Poole D, Chieregato A, Langer M, Viaggi B, Cingolani E, Malacarne P, et al. Systematic review of the literature and evidence-based recommendations for antibiotic prophylaxis in trauma: results from an Italian consensus of experts. PLoS One. 2014;9(11):e113676. DOI 10.1371/journal.pone.0113676.

72. Eftekhar B, Ghodsi M, Nejat F, Ketabchi E, EsmaeeIi B. Prophylactic administration of ceftriaxone for the prevention of meningitis after traumatic pneumocephalus: results of a clinical trial. J Neurosurg. 2004;101(5):757-61. DOI 10.3171/jns.2004.101.5.0757. 
73. Fang $C$, Zhu T, Zhang P, Xia L, Sun C. Risk factors of neurosurgical site infection after craniotomy: A systematic review and meta-analysis. Am J Infect Control. 2017;45(11):e123-e34. DOI 10.1016/j.ajic.2017.06.009.

74. Fei Q, Li J, Lin J, Li D, Wang B, Meng H, et al. Risk Factors for Surgical Site Infection After Spinal Surgery: A Meta-Analysis. World Neurosurg. 2016;95:507-15. DOI 10.1016/j.wneu.2015.05.059.

75. Zyck S, Toshkezi G, Krishnamurthy S, Carter DA, Siddiqui A, Hazama A, et al. Treatment of Penetrating Nonmissile Traumatic Brain Injury. Case Series and Review of the Literature. World Neurosurg. 2016;91:297-307. DOI 10.1016/j.wneu.2016.04.012.

76. Lazennec JY, Fourniols E, Lenoir T, Aubry A, Pissonnier ML, Issartel $\mathrm{B}$, et al. Infections in the operated spine: update on risk management and therapeutic strategies. Orthop Traumatol Surg Res. 2011;97(6 Suppl):S107-16. DOI 10.1016/j.otsr.2011.07.002.

77. Stevens DL, Bisno AL, Chambers HF, Dellinger EP, Goldstein EJ, Gorbach SL, et al. Practice guidelines for the diagnosis and management of skin and soft tissue infections: 2014 update by the infectious diseases society of America. Clin Infect Dis. 2014;59(2):147-59. DOI 10.1093/cid/ciu296.

78. Barker FG. Efficacy of Prophylactic Antibiotic Therapy in Spinal Surgery: A Meta-analysis. Neurosurgery. 2002;51(2):391-401.

79. Levi AD, Dickman CA, Sonntag VK. Management of postoperative infections after spinal instrumentation. J Neurosurg. 1997;86(6):975-80.

80. Massie J, Heller J, Abitbol J, McPherson D, Garfin S. Postoperative posterior spinal wound infections. Clin Orthop Relat Res 1992(284):99-108. DOI 10.3171/jns.1997.86.6.0975.

81. National Nosocomial Infections Surveillance System. National Nosocomial Infections Surveillance (NNIS) System Report, data summary from January 1992 through June 2004, issued October 2004. Am J Infect Control. 2004;32(8):470-85. DOI 10.1016/ S0196655304005425.

82. OIsen MA, Mayfield J, Lauryssen C, Polish L, al Jones $M$, Vest J. Risk factors for surgical site infection in spinal surgery. J Neurosurg. 2003;98(2):149-55. DOI 10.3171/spi.2003.98.2.0149.

83. Pawar AY, Biswas SK. Postoperative Spine Infections. Asian Spine J. 2016;10(1):176-83. DOI 10.4184/ asj.2016.10.1.176.
84. Rechtine GR, Bono PL, Cahill D, Bolesta MJ, Chrin AM. Postoperative Wound Infection after Instrumentation of Thoracic and Lumbar Fractures. J Orthop Trauma. 2001;15(8):566-9.

85. Stolke D, Sollmann W, Seifert $\nabla$. Intra- and postoperative complications in lumbar disc surgery. Spine (Phila Pa 1976) 1989;14(1):56-9.

86. Thalgott JS, Cotler HB, Sasso RC, LaRocca H, Gardner $\nabla$. Postoperative Infections in Spinal Implants Classification and Analysis-A Multicenter Study. Spine. 1991;16(8):981-4.

87. Weinstein MA, McCabe JP, Cammisa FP Postoperative Spinal Wound Infection: A Review of 2,391 Consecutive Index Procedures. J Spinal Disord. 2000;13(5):422-6.

88. Mitra A, Mitra A, Harlin S. Treatment of massive thoracolumbar wounds and vertebral osteomyelitis following scoliosis surgery. Plast Reconstr Surg. 2004;113(1):206-13.

89. Silber JS, Anderson DG, Vaccaro AR, Anderson PA, McCormick P, NASS. Management of postprocedural discitis. Spine J. 2002;2(4):279-87.

90. Pee YH, Park JD, Choi YG, Lee SH. Anterior debridement and fusion followed by posterior pedicle screw fixation in pyogenic spondylodiscitis: autologous iliac bone strut versus cage. J Neurosurg Spine. 2008;8(5):405-12. DOI 10.3171/SPI/2008/8/5/405

91. Sierra-Hoffman M, Jinadatha C, Carpenter JL, Rahm M. Postoperative instrumented spine infections: a retrospective review. South Med J. 2010;103(1):25-30. DOI 10.1097/SMJ.0b013e3181c4e00b.

92. Weiss L, Vaccaro A, Scuderi G, McGuire M, Garfin S. Pseudarthrosis after postoperative wound infection in the lumbar spine. J Spinal Disord. 1997;10(6):482-7.

93. Gruenberg MF, Campaner GL, Sola CA, Ortolan EG. Ultraclean Air for Prevention of Postoperative infection after posterior spinal fusion with instrumentation. Spine. 2004;29(20):2330-4.

94. Viola R, King H, Adler S, Wilson C. Delayed infection after elective spinal instrumentation and fusion. A retrospective analysis of eight cases. Spine (Phila Pa 1976). 1997;22:2444-50.

95. Watt JP, Dunn RN. The Use of Vacuum Dressings for Dead Space Management in Deep Surgical Site Infections Allows Implant and Bone Graft 
Retention. Global Spine J. 2017;7(8):756-61. DOI 10.1177/2192568217696694.

96. Liu C, Bayer A, Cosgrove SE, Daum RS, Fridkin SK, Gorwitz RJ, et al. Clinical practice guidelines by the infectious diseases society of america for the treatment of methicillin-resistant Staphylococcus aureus infections in adults and children. Clin Infect Dis. 2011;52(3):e18-55. DOI 10.1093/cid/ciq146.

97. Bryant M, Bremer A, Tepas Jr, Mollitt D, Nquyen T, Talbert J. Abdominal complications of ventriculoperitoneal shunts. Case reports and review of the literature. Am Surg. 1988;54:50-5.

98. Mayhall CG, Archer NH, Lamb VA, Spadora AC, Baggett JW, Ward JD, et al. Ventriculostomy-related infections. A prospective epidemiologic study. N Engl J Med. 1984;310(9):553-9. DOI 10.1056/ NEJM198403013100903.

99. Odio C, McCracken GJ, Nelson J. CSF shunt infections in pediatrics. A seven- year experience. Am J Dis Child. 1984;138(12):1103-8.

100. Van de Beek D, Drake JM, Tunkel AR. Nosocomial bacterial meningitis. N Engl J Med. 2010;362(2):14654. DOI 10.1056/NEJMra0804573.

101. Forgacs P, Geyer CA, Freidberg SR. Characterization of chemical meningitis after neurological surgery. Clin Infect Dis. 2001;32(2):179-85. DOI 10.1086/318471.

102. Schade RP, Schinkel J, Roelandse FW, Geskus RB, Visser LG, van Dijk JM, et al. Lack of value of routine analysis of cerebrospinal fluid for prediction and diagnosis of external drainage-related bacterial meningitis. J Neurosurg. 2006;104(1):101-8. DOI 10.3171/jns.2006.104.1.101

103. Xiao X, Zhang Y, Zhang L, Kang P, Ji N. The diagnostic value of cerebrospinal fluid lactate for post-neurosurgical bacterial meningitis: a meta-analysis. BMC Infect Dis. 2016;16:483. DOI 10.1186/s12879-016-1818-2.

104. Tunkel AR, Hartman BJ, Kaplan SL, Kaufman BA, Roos KL, Scheld WM, et al. Practice guidelines for the management of bacterial meningitis. Clin Infect Dis. 2004;39(9):1267-84. DOI 10.1086/425368.

105. Antony SJ, Hoffman-Roberts HL, Foote BS. Use of Daptomycin as Salvage Therapy in the Treatment of Central Nervous System Infections Including Meningitis and Shunt Infections. Infectious Diseases in Clinical Practice. 2012;20(2):161-3.
106. Dunne WM, Mason EO, Kaplan SL. Diffusion of rifampin and vancomycin through a Staphylococcus epidermidis biofilm. Antimicrobial Agents and Chemotherapy. 1993;37(12):2522-6.

107. Gombert ME, Landesman SH, Corrado ML, Stein SC, Melvin ET, Cummings M. Vancomycin and rifampin therapy for Staphylococcus epidermidis meningitis associated with CSF shunts: report of three cases. J Neurosurg. 1981;55(4):633-6. DOI 10.3171/ jns.1981.55.4.0633.

108. Groll AH, Giri N, Petraitis V, Petraitiene R, Candelario M, Bacher JS, et al. Comparative efficacy and distribution of lipid formulations of amphotericin B in experimental Candida albicans infection of the central nervous system. J Infect Dis. 2000;182(1):274-82. DOI $10.1086 / 315643$.

109. Kim B-N, Peleg AY, Lodise TP, Lipman J, Li J, Nation $\mathrm{R}$, et al. Management of meningitis due to antibioticresistant Acinetobacter species. Lancet Infect Dis. 2009;9(4):245-55. DOI 10.1016/S1473-3099(09)70055-6.

110. Milstone AM, Dick J, Carson B, Siberry GK. Cerebrospinal fluid penetration and bacteriostatic activity of linezolid against Enterococcus faecalis in a child with a ventriculoperitoneal shunt infection. Pediatr Neurosurg. 2007;43(5):406-9. DOI 10.1159/000106392.

111. Pappas PG, Kauffman CA, Andes DR, Clancy CJ, Marr KA, Ostrosky-Zeichner L, et al. Clinical Practice Guideline for the Management of Candidiasis: 2016 Update by the Infectious Diseases Society of America. Clin Infect Dis. 2016;62(4):e1-50. DOI 10.1093/cid/ciø933.

112. Patterson TF, Thompson GR 3rd, Denning DW, Fishman JA, Hadley S, Herbrecht R, et al. Practice Guidelines for the Diagnosis and Management of AspergiIlosis: 2016 Update by the Infectious Diseases Society of America. Clin Infect Dis. 2016;63(4):e1-e60. DOI 10.1093/cid/ciw326.

113. ybak M, Lomaestro B, Rotschafer JC, Moellering R, Jr, Craig W, Billeter M, et al. Therapeutic monitoring of vancomycin in adult patients: a consensus review of the American Society of Health-System Pharmacists, the Infectious Diseases Society of America, and the Society of Infectious Diseases Pharmacists. Am J Health Syst Pharm. 2009;66(1):82-98. DOI 10.2146/ ajhp080434.

114. Vilmaz A, Dalgic N, Musluman M, Sancar M, Colak I, Aydin Y. Linezolid treatment of shunt-related cerebrospinal fluid infections in children. J Neurosurg 
Pediatr. 2010;5(5):443-8. DOI 10.3171/2009.12. PEDS09421.

115. Kestle JR, Garton HJ, Whitehead WE, Drake JM, Kulkarni AV, Cochrane DD, et al. Management of shunt infections: a multicenter pilot study. J Neurosurg. 2006;105(3 SuppI):177-81. DOI 10.3171/ ped.2006.105.3.177.

116. James H, Walsh J, Wilson H, Connor J. The management of cerebrospinal fluid shunt infections: a clinical experience. Acta Neurochir (Wien). 1981;59:157-66.

117. Schreffler RT, Schreffler AJ, Wittler RR. Treatment of cerebrospinal fluid shunt infections: a decision analysis. Pediatr Infect Dis J. 2002;21(7):632-6. DOI 10.1097/01.inf.0000020964.43766.35.

118. Yogev R. Cerebrospinal fluid shunt infections: a personal view. Pediatr Infect Dis. 1985;4(2):113-8.

119. Whitehead WE, Kestle JR. The treatment of cerebrospinal fluid shunt infections. Results from a practice survey of the American Society of Pediatric Neurosurgeons. Pediatr Neurosurg. 2001;35(4):205-10. DOI 10.1159/000050422.

120. Chapman P, Borges L. Shunt infections: prevention and treatment. Clin Neurosuro. 1985;32:652-64.

121. Brown EM, Edwards RJ, Pople IK. Conservative management of patients with cerebrospinal fluid shunt infections. Neurosurgery. 2006;58(4):657-65; discussion -65. DOI 10.1227/01.NEU.0000204126.54417.46.

122. Brown J, de Louvois R, Bayston PD, Lees IK, Pople EM. The management of neurosurgical patients with postoperative bacterial or aseptic meningitis or external ventricular drain-associated ventriculitis. British journal of neurosurgery. 2009;14(1):7-12.

123. Tamber MS, Klimo P. Jr, Mazzola CA, Flannery AM, Pediatric Hydrocephalus Systematic R, EvidenceBased Guidelines Task F. Pediatric hydrocephalus: systematic literature review and evidence-based guidelines. Part 8: Management of cerebrospinal fluid shunt infection. J Neurosurg Pediatr. 2014;14 (Suppl 1:)60-71. DOI 10.3171/2014.7.PEDS14328.

124. Wen DY, Bottini AG, Hall WA, Haines SJ. Infections in neurologic surgery. The intraventricular use of antibiotics. Neurosurg Clin N Am. 1992;3:343-54. DOI 10.1016/S1042-3680(18)30666-1.

125. Wilkie MD, Hanson MF, Statham PF, Brennan PM. Infections of cerebrospinal fluid diversion devices in adults: the role of intraventricular antimicrobial therapy. J Infect. 2013;66(3):239-46. DOI 10.1016/j. jinf.2012.11.006.

126. Shah SS, OhIsson A, Shah VS. Intraventricular antibiotics for bacterial meningitis in neonates. Cochrane Database Syst Rev. 2012(7):CD004496. DOI 10.1002/14651858.CD004496.pub3.

127. Fu RZ, Anwar DR, Laban JT, Maratos EC, Minhas PS, Martin AJ. Pre-emptive intrathecal vancomycin therapy reduces external ventricular drain infection: a single centre retrospective case-control study. Br J Neurosurg. 2017;31(1):16-20. DOI 10.1080/02688697.2016.1229741.

128. Chen K, Wu Y, Wang Q, Wang J, Li X, Zhao Z, et al. The methodology and pharmacokinetics study of intraventricular administration of vancomycin in patients with intracranial infections after craniotomy. J Crit Care. 2015;30(1):218 e1-5. DOI 10.1016/j.jcrc.2014.09.020.

129. Cook AM, Mieure KD, Owen RD, Pesaturo AB, Hatton $\mathrm{J}$. Intracerebroventricular administration of drugs. Pharmacotherapy. 2009;29(7):832-45. DOI 10.1592/ phco.29.7.832.

130. Grill MF, Maganti RK. Neurotoxic effects associated with antibiotic use: management considerations. Br J Clin Pharmacol. 2011;72(3):381-93. DOI 10.1111/j.1365-2125.2011.03991.x.

131. Imberti R, Cusato M, Accetta G, Marino V, Procaccio F, Del Gaudio A, et al. Pharmacokinetics of colistin in cerebrospinal fluid after intraventricular administration of colistin methanesulfonate. Antimicrob Agents Chemother. 2012;56(8):4416-21. DOI 10.1128/ AAC.00231-12.

132. LeBras M, Chow I, Mabasa VH, Ensom MH. Systematic Review of Efficacy, Pharmacokinetics, and Administration of Intraventricular Aminoglycosides in Adults. Neurocrit Care. 2016;25(3):492-507. DOI 10.1007/s12028-016-0269-3.

133. Nau R, Seele J, Djukic M, Eiffert H. Pharmacokinetics and pharmacodynamics of antibiotics in central nervous system infections. Curr Opin Infect Dis. 2018;31(1):57-68. DOI 10.1097/QCO.00000000000000418.

134. Ng K, Mabasa VH, Chow I, Ensom MH. Systematic review of efficacy, pharmacokinetics, and administration of intraventricular vancomycin in adults. Neurocrit Care. 2014;20(1):158-71. DOI 10.1007/ s12028-012-9784-z. 
135. Pfausler B, Haring H, Kampfl A, Wissel J, Schober M, Schmutzhard E. Cerebrospinal fluid (CSF) pharmacokinetics of intraventricular vancomycin in patients with staphylococcal ventriculitis associated with external CSF drainage. Clin Infect Dis. 1997;25:733-5.

136. Cruciani M, Navarra A, Di Perri G, Andreoni M, Danzi M, Concia E. Evaluation of intraventricular teicoplanin for the treatment of neurosurgical shunt infections. Clin Infect Dis. 1992;15:285-9.

137. Elvy J, Porter D, Brown E. Treatment of external ventricular drain-associated ventriculitis caused by Enterococcus faecalis with intraventricular daptomycin. J Antimicrob Chemother. 2008;61(2):461-2. DOI 10.1093/jac/dkm501.

138. Erritouni M, Ktaich N, Rahal JJ, Figueroa D, Nieto J, Urban $C$, et al. Use of daptomycin for the treatment of methicillin-resistant coagulase-negative staphylococcal ventriculitis. Case Rep Med. 2012;2012:593578. DOI 10.1155/2012/593578.

139. Falagas ME, Bliziotis IA, Tam VH. Intraventricular or intrathecal use of polymyxins in patients with Gramnegative meningitis: a systematic review of the available evidence. Int J Antimicrob Agents. 2007;29(1):925. DOI 10.1016/j.ijantimicag.2006.08.024.

140. Jaspan HB, Brothers AW, Campbell AJ, McGuire JK, Browd SR, Manley TJ, et al. Multidrug-resistant Enterococcus faecium meningitis in a toddler: characterization of the organism and successful treatment with intraventricular daptomycin and intravenous tigecycline. Pediatr Infect Dis J. 2010;29(4):379-81. DOI 10.1097/INF.0b013e3181c806d8.

141. Katragkou A, Roilides E. Successful treatment of multidrug-resistant Acinetobacter baumannii central nervous system infections with colistin. J Clin Microbiol. 2005;43(9):4916-7. DOI 10.1128/ JCM.43.9.4916-4917.2005.

142. Mueller SW, Kiser TH, Anderson TA, Neumann RT. Intraventricular daptomycin and intravenous linezolid for the treatment of external ventriculardrain-associated ventriculitis due to vancomycinresistant Enterococcus faecium. Ann Pharmacother. 2012;46(12):e35. DOI 10.1345/aph.1R412.

143. Pfausler B, Spiss H, Beer R, Kampl A, Engelhardt $\mathrm{K}$, Schober $M$, et al. Treatment of staphylococcal ventriculitis associated with external cerebrospinal fluid drains: a prospective randomized trial of intravenous compared with intraventricular vancomycin therapy. J Neurosurg. 2003;98(5):1040-4. DOI 10.3171/jns.2003.98.5.1040.

144. Rodriguez Guardado A, Blanco A, Asensi V, Perez F, Rial JC, Pintado $\nabla$, et al. Multidrug-resistant Acinetobacter meningitis in neurosurgical patients with intraventricular catheters: assessment of different treatments. J Antimicrob Chemother. 2008;61(4):90813. DOI 10.1093/jac/dkno18.

145. Tangden T, Enblad P, Ullberg M, Sjolin J. Neurosurgical gram-negative bacillary ventriculitis and meningitis: a retrospective study evaluating the efficacy of intraventricular gentamicin therapy in 31 consecutive cases. Clin Infect Dis. 2011;52(11):1310-6. DOI 10.1093/cid/cir 197 .

146. Wang JH, Lin PC, Chou CH, Ho CM, Lin KH, Tsai CT, et al. Intraventricular antimicrobial therapy in postneurosurgical Gram-negative bacillary meningitis or ventriculitis: a hospital-based retrospective study. J Microbiol Immunol Infect. 2014;47(3):204-10. DOI 10.1016/j.jmii.2012.08.028.

147. Williamson JC, Glazier SS, Peacock JE. Successful treatment of ventriculostomy-related meningitis caused by vancomycin-resistant Enterococcus with intravenous and intraventricular quinupristin/dalfopristin. Clin Neurol Neurosurg. 2002;104(1):54-6.

148. Ziai WC, Lewin JJ. Improving the role of intraventricular antimicrobial agents in the management of meningitis. Curr Opin Neurol. 2009;22(3):277-82.

149. Savitz SI, Rivlin MM, Savitz MH. The ethics of prophylactic antibiotics for neurosurgical procedures. J Med Ethics. 2002;28(6):358-63.

150. Attenello FJ, Garces-Ambrossi GL, Zaidi HA, Sciubba DM, Jallo GI. Hospital costs associated with shunt infections in patients receiving antibiotic-impregnated shunt catheters versus standard shunt catheters. Neurosurgery. 2010;66(2):284-9; discussion 9. DOI 10.1227/01.NEU.0000363405.12584.4D.

151. Eder C, Schenk S, Trifinopoulos J, Kulekci B, KienzI M, Schildbock S, et al. Does intrawound application of vancomycin influence bone healing in spinal surgery? Eur Spine J. 2016;25(4):1021-8.

152. Edwards NC, Engelhart L, Casamento EM, McGirt MJ. Cost-consequence analysis of antibiotic-impregnated shunts and external ventricular drains in hydrocephalus. Journal of neurosurgery. 2015;122(1):13947. DOI 10.1007/s00586-015-3943-9. 
153. Eymann R, Chehab S, Strowitzki M, SteudeI WI, Kiefer M. Clinical and economic consequences of antibiotic-impregnated cerebrospinal fluid shunt catheters. J Neurosurg Pediatr. 2008;1(6):444-50. DOI 10.3171/PED/2008/1/6/444.
154. Lonsdale DO, Udy AA, Roberts JA, Lipman J. Antibacterial therapeutic drug monitoring in cerebrospinal fluid: difficulty in achieving adequate drug concentrations. Journal of neurosurgery. 2013;118(2):297301. DOI 10.3171/2012.10.JNS12883. 